International Joumal of Biological Sciences

2009; 5(5):428-437

Research Paper

(C) Ivyspring International Publisher. All rights reserved

\title{
Nuclear Localization of p38 MAPK in Response to DNA Damage
}

\section{David Wood'1, Tina M. Thornton'1, Guadalupe Sabio², Roger A. Davis ${ }^{2}$, and Mercedes Rincon ${ }^{1 凶}$}

1. Department of Medicine/Immunobiology Program, University of Vermont, Burlington, VT 05405. USA.

2. Program in Molecular Medicine, University of Massachusetts, Worcester, MA 01605. USA.

$\triangle$ Correspondence to: Mercedes Rincon, tel: +1 802656 0937; fax: +1 802656 3854; email: mercedes.rincon@uvm.edu Received: 2009.04.30; Accepted: 2009.06.10; Published: 2009.06.16

\begin{abstract}
P38 MAP kinase (MAPK) is activated in response to environmental stress, cytokines and DNA damage, and mediates death, cell differentiation and cell cycle checkpoints. The intracellular localization of p38 MAPK upon activation remains unclear, and may depend on the stimulus. We show here that activation of p38 MAPK by stimuli that induce DNA double strand breaks (DSBs), but not other stimuli, leads to its nuclear translocation. In addition, naturally occurring DSBs generated through V(D)J recombination in immature thymocytes also promote nuclear accumulation of p38 MAPK. Nuclear translocation of p38 MAPK does not require its catalytic activity, but is induced by a conformational change of p38 MAPK triggered by phosphorylation within the active site. The selective nuclear accumulation of p38 MAPK in response to DNA damage could be a mechanism to facilitate the phosphorylation of p38 MAPK nuclear targets for the induction of a G2/M cell cycle checkpoint and DNA repair.
\end{abstract}

Key words: p38 MAP kinase, DNA damage, cell cycle checkpoint, DNA repair

\section{Introduction}

The p38 MAPK family are a group of kinases belonging to the MAPK family, together with the JNK and ERK groups. There are four different isoforms of p38 MAPK (p38 $\alpha, p 38 \beta, p 38 \gamma$, and p38 $)$. p38 $\alpha$ is the most abundant and widely expressed, but it is also the only isoform with a non-redundant function in vivo [1-5]. p38 MAPK is activated following phosphorylation at $\mathrm{Thr}^{180} / \mathrm{Tyr}^{182}$ within the active site. This phosphorylation is mediated primarily by upstream MKK3 and MKK6, although in vitro, MKK4 may also contribute to p38 MAPK activation in the absence of MKK3/MKK6 [6-8]. MKK3 and MKK6 in turn are regulated by phosphorylation through upstream MAPK kinase kinases (MAPKKK).

The p38 MAPK pathway is activated in response to environmental stress (UV, ionizing radiation, oxidative stress, and FAS ligand) and cytokines (e.g. TNFo) [9]. Activation of the p38 MAPK pathway by cytokines and receptor ligands, normally leads to cell differentiation. Activation of p38 MAPK through environmental stress can mediate cell death. In response to DNA damage stimuli that induce DSBs (ionizing radiation, UV, chemotherapeutic drugs) activation of p38 MAPK can also lead to the induction of a G2/M cell cycle checkpoint through p53-dependent and independent mechanisms [10-15]. p38 MAPK can also be activated by naturally generated DSBs in immature thymocytes at the CD4-CD8- double negative $(\mathrm{DN}) 3$ stage, whilst they undergo $\mathrm{V}(\mathrm{D}) \mathrm{J}$ recombination of the T cell receptor (TCR) $\beta$ gene.

Despite the number of studies showing activation of p38 MAPK in response to DNA damage inducing stimuli and its role in the G2/M checkpoint, the mechanism by which p38 MAPK is activated, and its intracellular distribution is unclear. Unlike other MAPK, p38 MAPK has no nuclear localization signal, and has been shown to be distributed throughout the cytosol and nucleus [16]. However, in response to 
specific stimuli p38 MAPK has been shown to preferentially accumulate in the cytosol [17]. Depending on the stimuli, p38 MAPK can have a variety of substrates, including transcription factors (ATF2, MEF2), protein kinases (MnK, MAPKAPK2), death/survival molecules (Bcl2, caspases), and cell cycle control factors (cyclin D1) [18-22]. Thus, it is possible that the intracellular distribution of p38 MAPK is associated with its substrate specificity and determined by the nature of the stimuli.

We show here that p38 MAPK translocates to the nucleus specifically upon activation by stimuli that induce DSBs, and that p38 MAPK nuclear translocation is triggered by a conformational change induced by phosphorylation within the active site. This specific nuclear translocation could be relevant for this pathway to regulate the initiation of a G2/M cell cycle checkpoint and promote DNA repair.

\section{Results}

\section{DNA Damage Induces the Nuclear Transloca- tion of p38 MAPK.}

Despite its role in the induction of cell cycle checkpoints in response to DSBs inducing stimuli, little is known about the intracellular distribution of p38 MAPK following its activation by DSBs. Unlike other MAPKs, p38 MAPK lacks a specific nuclear localization sequence. We therefore investigated the intracellular localization of p38 MAPK in response to UV radiation, which induces DNA single strand damage that can lead to the formation of DNA DSBs [23]. 293T cells were transfected with p38 MAPK and exposed to UV irradiation. Cells were stained for $\mathrm{p} 38$ MAPK and examined by confocal microscopy. In unexposed cells, p38 MAPK was distributed throughout the cell, but predominantly outside the nucleus (Fig 1A). Interestingly, following exposure to UV, p38 MAPK accumulated in the nucleus (Fig 1A). The generation of DSBs in response to UV exposure was monitored by staining cells for the presence of phosphorylated H2AX at Ser139 (termed $\gamma \mathrm{H} 2 \mathrm{AX}$ ), a known indicator of DNA DSBs (Fig 1A) [24, 25]. To corroborate that this nuclear translocation was due to the presence of DSBs, cells were exposed to $X$-radiation, a known source of ionizing radiation that induces DNA DSBs. Similar to UV exposure, $X$-radiation also caused a translocation of p38 MAPK to the nucleus (Fig 1A). To visualize phospho-p38 in this study, an antibody that recognizes p38 MAPK which has been dually phosphorylated on both $\mathrm{Thr}^{180} / \mathrm{Tyr}^{182}$ was used. Staining for phospho-p38 MAPK showed no phosphorylation of p38 MAPK in unexposed cells (Fig 1B), but high levels of phos- phorylated p38 MAPK specifically in the nuclei of cells exposed to either UV or X-radiation (Fig 1B). Thus, nuclear localization of p38 MAPK in response to DNA damage inducing stimuli is associated with its phosphorylation.

Since a nuclear translocation of p38 MAPK upon cell stimulation has not been previously reported, we examined the distribution of p38 MAPK in response to other known activators which do not induce DNA damage. Treatment with TNF $\alpha$, one of the first identified activators of p38 MAPK, did not cause p38 MAPK nuclear accumulation (Fig 1C), although p38 MAPK phosphorylation was induced (Fig 1D). Similarly, Fas ligation or PMA activated p38 MAPK (Fig 1D), but did not promote its nuclear accumulation (Fig 1C). Thus, p38 MAPK is selectively translocated to the nucleus in response to stimuli that generate DSBs.

\section{Phosphorylation of p38 MAPK is Essential for its Nuclear Translocation in Response to DNA Damage.}

p38 MAPK is activated following phosphorylation at $\mathrm{Thr}^{180} / \mathrm{Tyr}^{182}$ by upstream MAPKKs [16]. In order to assess whether phosphorylation of p38 MAPK was required for its nuclear translocation following DNA damage, cells were transfected with either wild type p38 MAPK or a dominant negative p38 MAPK (dnp38) mutant where Thr ${ }^{180} / \mathrm{Tyr}^{182}$ have been substituted with non-phosphorylatable Ala/Phe residues respectively [16]. Whilst wild type p38 MAPK was distributed throughout the cells, dnp38 was totally excluded from the nucleus in untreated cells (Fig 2A). In contrast to wild type p38 MAPK which accumulated preferentially in the nucleus, dnp38 remained in the cytosol and was excluded from the nucleus following exposure to $\mathrm{X}$-radiation (Fig 2A). The presence of $\gamma \mathrm{H} 2 \mathrm{AX}$ showed that cells transfected with dnp38 have DSBs, indicating the activation of the DNA damage response (Fig 2A). Similar to X-radiation, UV exposure failed to induce nuclear translocation of dnp38 (Fig 2B). Thus, phosphorylation of $\mathrm{p} 38 \mathrm{MAPK}$ at $\mathrm{Thr}^{180} / \mathrm{Tyr}^{182}$ is required for the nuclear translocation of p38 MAPK following DNA damage.

Phosphorylation of p38 MAPK leads to its activation by inducing a conformational change. To dissociate whether p38 MAPK activity, or solely the conformational change caused by the phosphorylation was required for nuclear translocation we examined the effect of a pharmacological p38 MAPK inhibitor, SB203580 [26], which binds at the catalytic site and blocks its activity. Cells were transfected 
with wild type p38 MAPK and pretreated with SB203580 prior to $X$-radiation exposure. Interestingly, SB203580 did not prevent nuclear translocation of p38 MAPK in response to X-radiation (Fig 2C). Similarly, no effect of SB203580 on UV-induced nuclear translocation of p38 MAPK could be detected (Fig 2D).
Thus, catalytic activity of p38 MAPK is not required for its nuclear translocation in response to DNA damage, suggesting that a conformational change caused by upstream phosphorylation promotes its nuclear transport.
A

\section{Unstim}

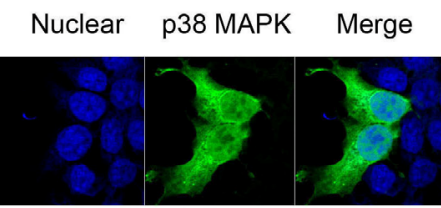

U.V.
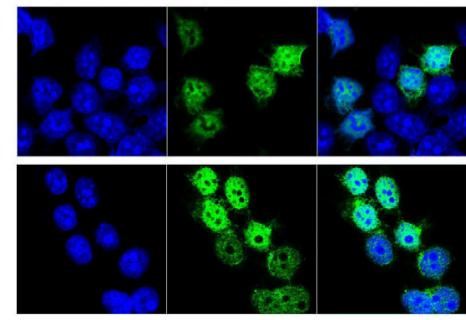

C

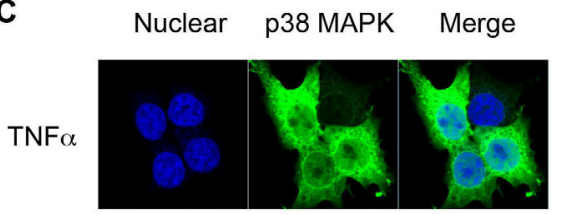

FAS-L

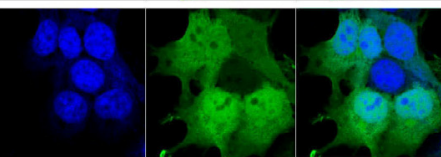

PMA

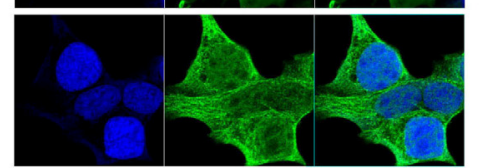

$\gamma-\mathrm{H} 2 \mathrm{AX}$
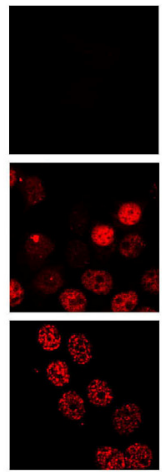

$\gamma-\mathrm{H} 2 \mathrm{AX}$
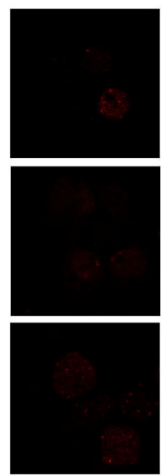

B

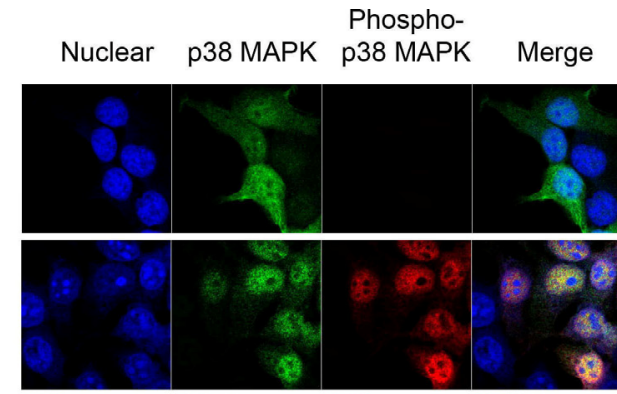

X-Ray

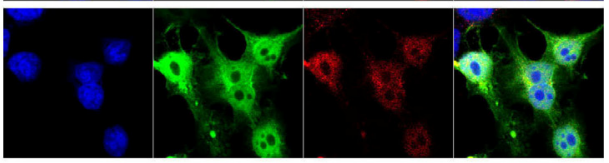

Phospho-

Nuclear p38 MAPK p38 MAPK Merge

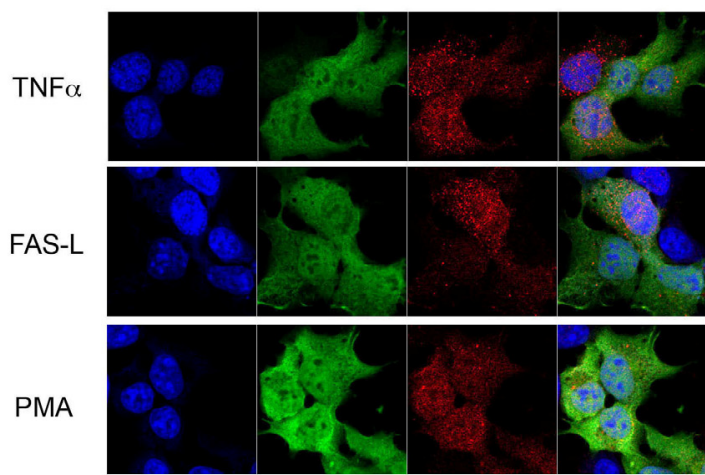

Figure I. Nuclear Translocation of p38 MAPK in Response to DNA Damage Stimuli. (A) 293T cells were transiently transfected with wild type P38 MAPK and left unstimulated (unstim), or treated with UV irradiation (UV), or X-radiation (X-Ray). Cells were stained for p38 MAPK (green) and $\gamma \mathrm{H} 2 \mathrm{AX}$ (red). TOPRO-3 (blue) was used as a nuclear marker. (B) 293T cells were transfected and treated as in (A). Cells were stained for p38 MAPK (green), and phospho-p38 MAPK (red). 'Merge' indicates the merged images of TOPRO-3 and p38 MAPK or TOPRO-3, p38 MAPK, and phospho-p38 MAPK. (C) and (D) 293T transfected cells as in (A) were treated with TNF $\alpha(10 \mathrm{ng} / \mathrm{ml}), \mathrm{lg}$-FASL $(200 \mathrm{ng} / \mathrm{ml})$ and cross linker $(4 \mu \mathrm{g} / \mathrm{ml})$, or PMA (10 ng/ml) for 30 mins. Staining and confocal analysis were performed as described in (A). Data representative of at least three independent experiments. 

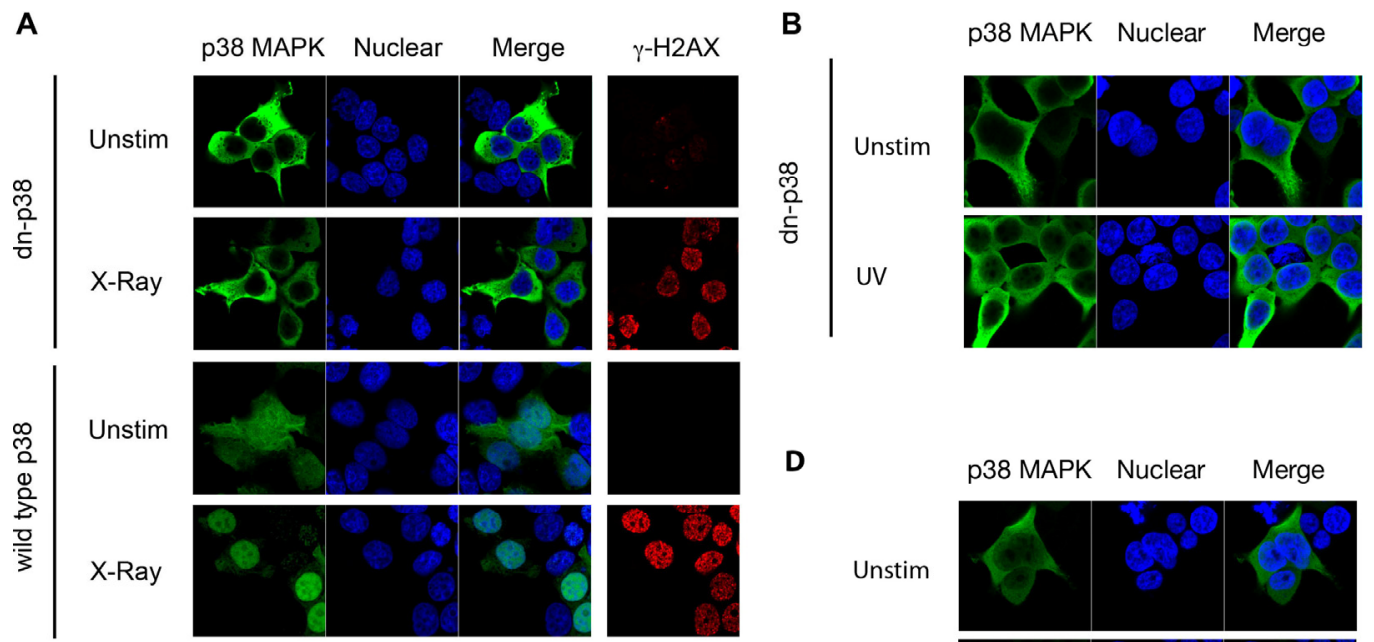

YH2AX
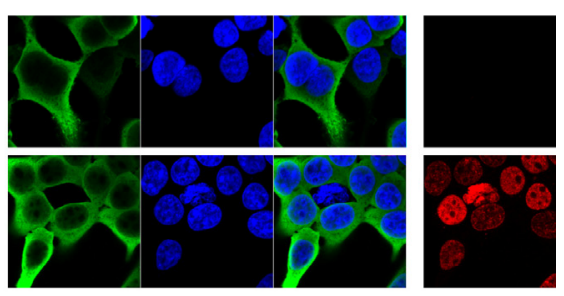

D
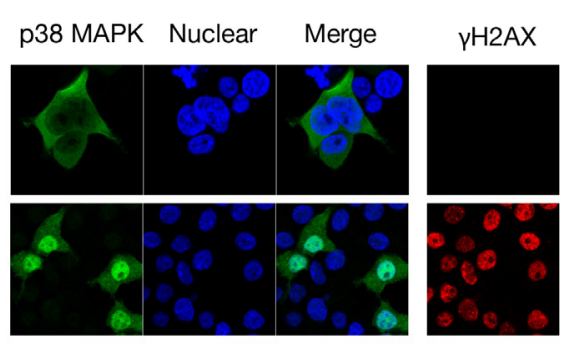

C
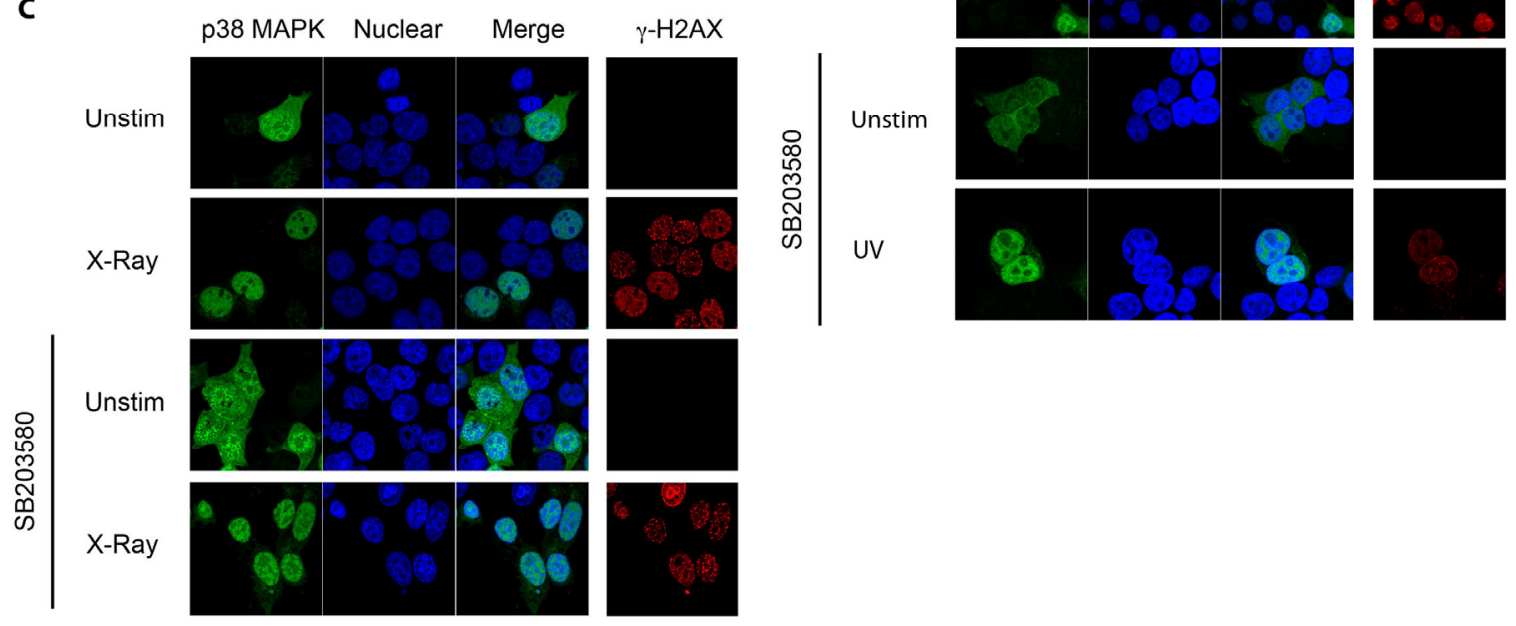

Figure 2. p38 MAPK Phosphorylation is Required for its Nuclear Translocation. (A) 293T cells were transiently transfected with either wild type or dnp38 MAPK and left unstimulated (unstim), or treated with X-Ray. Cells were stained for p38 MAPK (green) and $\gamma \mathrm{H} 2 \mathrm{AX}$ (red). TOPRO-3 (blue) was used as a nuclear marker. (B) 293T cells were transfected with dnp38 MAPK and left unstimulated (unstim), or treated with UV irradiation (UV). Staining and confocal analysis were performed as described in (A). (C) and (D) 293T cells transiently transfected with wild type p38 MAPK, were treated with SB203580 (5 $\mu \mathrm{M}) 40$ mins, and left unstimulated or exposed to X-Ray (C) or UV (D). Staining and confocal analysis were performed as described in (A). 'Merge' indicates the merged images of TOPRO-3 and p38 MAPK. Data representative of at least three independent experiments.

Unlike SB203580, BIRB 796 belongs to a new class of p38 MAPK inhibitors, inhibiting p38 $\alpha$ MAPK via a novel mechanism which indirectly competes with ATP binding by inducing a conformational change [27]. Binding of BIRB 796 to p38 MAPK causes a large structural reorganization of the activation loop (residues 171-184) and the resulting structure is non-compatible with ATP-binding. This conformational change can resemble that induced by phosphorylation at $\mathrm{Thr}^{180} / \mathrm{Tyr}^{182}$. Since SB203580 did not affect p38 MAPK translocation, despite inhibiting p38 MAPK catalytic activity, we investigated whether a conformational change induced by BIRB 796 could affect the localization of the dnp38 mutant by mimicking the effect of phosphorylation. Cells were transfected with dnp38 and pretreated with BIRB 796 prior to UV exposure. Although dnp38 remained in the cytosol, the presence of BIRB 796 restored its nuclear translocation (Fig 3A). In contrast, dnp38 did not translocate to the nucleus in the presence of SB203580 (Fig 3A). To show that both compounds were able to inhibit the activity of p38 MAPK in these cells, we examined downstream targets of p38 MAPK. We analyzed the activation of Mnk kinase 
since it is a primary target for p38 MAPK but not for JNK [19]. Mnk1/2 mediate phosphorylation and activation of the translation initiator factor $4 \mathrm{E}$ (eIF-4E), and phosphorylation of eIF-4E is usually used as a read out system for activity of Mnk [19]. eIF-4E phosphorylation was induced upon UV exposure, but the presence of either SB203580 or BIRB 796 blocked its phosphorylation (Fig 3B). Thus, both pharmacological compounds are able to interfere with the catalytic activity of p38 MAPK. We also examined the effect of both inhibitors on the phosphorylation of p38 MAPK at the $\mathrm{Thr}^{180} / \mathrm{Tyr}^{182}$ motif by upstream MAPKK. According to its mechanism of action, SB203580 did not interfere with the phosphorylation of p38 MAPK (Fig 3C). In contrast, BIRB 796 reduced the phosphorylation of p38 MAPK at this domain, supporting the conformational change effect that this compound has (Fig 3C). Analysis of p38 MAPK cellular localization in non-UV exposed cells treated with BIRB 796, indicated that BIRB 796 by itself was also able to promote accumulation of dnp38 to the nucleus in the absence of other stimuli (Fig 3D). This further supports that p38 MAPK activation loop phosphorylation is critical to its nuclear translocation by inducing a conformational change.

\section{Nuclear Translocation of Endogenous p38 MAPK in Thymocytes Following Ionizing Radiation.}

To show that nuclear accumulation of endogenous p38 MAPK in response to ionizing radiation also occurs in primary cells, we examined endogenous p38 MAPK distribution in thymocytes. Total thymocytes from wild type mice were exposed to X-radiation, and stained for endogenous p38 MAPK. The intracellular distribution of p38 MAPK was examined by confocal microscopy. Unlike 293T cells, thymocytes have a very limited amount of cytoplasm (immediately surrounding the nucleus). Nonetheless, p38 MAPK could be detected in the cytosol of thymocytes prior to exposure (Fig 4A). However, exposure to ionizing radiation resulted in a nuclear accumulation of p38 MAPK (Fig 4A). These results further demonstrate that p38 MAPK specifically translocates to the nucleus in response to DNA damage.
A

UV
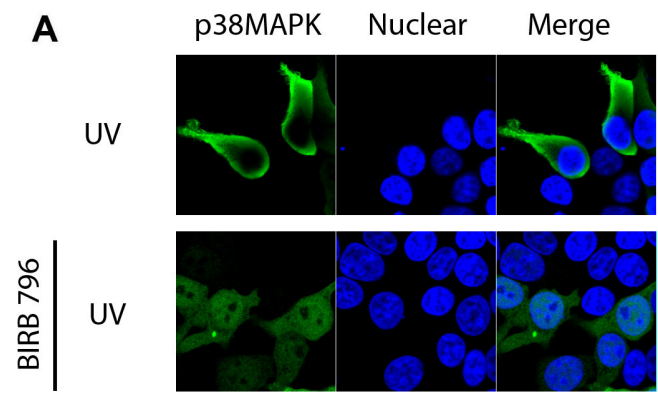

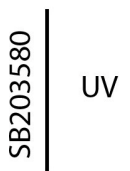

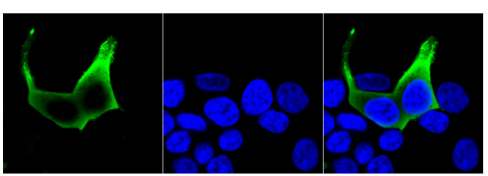

B

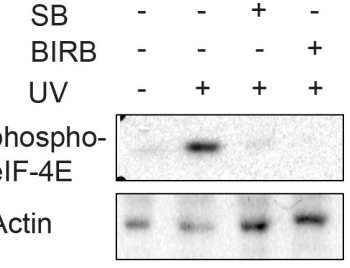

D

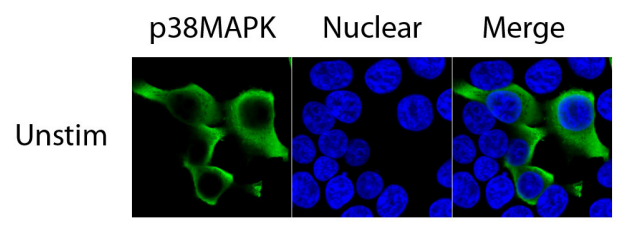

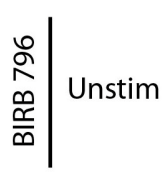

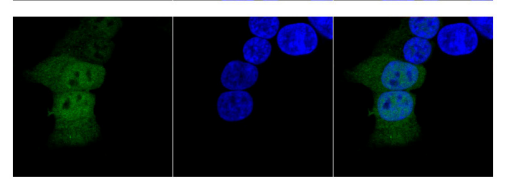

Figure 3. p38 MAPK Phosphorylation Induces a Conformational Change that is Required for Nuclear Translocation. (A) 293T cells transfected with dnp38 MAPK were treated with BIRB 796 (5 $\mu$ M) 40 mins, and exposed to UV irradiation (UV). Cells were stained for P38 MAPK (green) and TOPRO-3 (blue). 'Merge' indicates the merged images of TOPRO-3 and P38 MAPK. (B) 293T cells transiently transfected with wild type P38 MAPK, were left unstimulated or exposed to UV in the presence or absence of SB203580 (5 $\mu \mathrm{M})$ or BIRB $796(5 \mu \mathrm{M})$. Whole cell extracts were then examined for phosphorylated elF-4E by Western blot analysis. Actin levels were examined as a loading control. (C) Cells were exposed and treated as described in (B) and whole cell extracts were used to examine phospho-p38 MAPK and total p38 MAPK by Western blot analysis. (D) 293T cells transfected with dnp38 MAPK were treated with vehicle or BIRB 796 for 40 mins. Cells were stained as described in (A). Data representative of at least three independent experiments. 


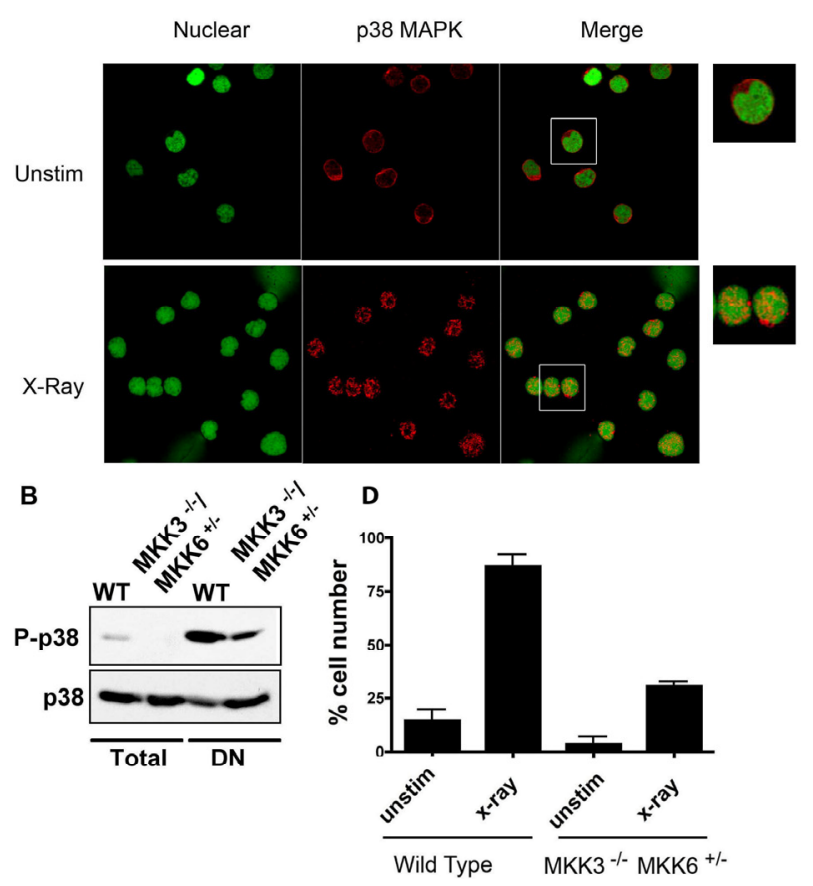

C

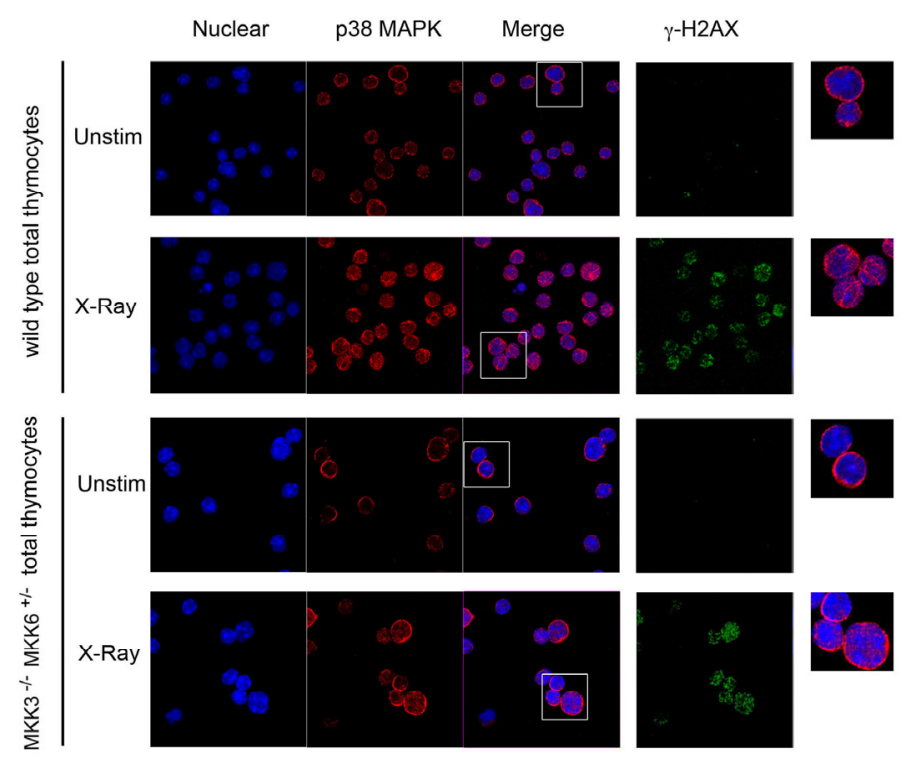

Figure 4. Nuclear Translocation of Endogenous P38 MAPK in Thymocytes in Response to DNA Damage. (A) Total thymocytes from wild type mice were left unstimulted (unstim), or exposed to X-Ray, and stained for p38 MAPK (red) and YoYo (green). (B) Whole cell extracts from total and purified DN thymocytes from wild type and $\mathrm{MKK}^{-1 /} / \mathrm{MKK6}^{+/-}$mice were examined for phospho-p38 MAPK and p38 MAPK by western blot analysis (C) Total thymocytes from both wild type and $\mathrm{MKK}^{-1-} / \mathrm{MKK}^{+/-}$mice were left unstimulted (unstim), or treated with $\mathrm{X}$-Ray, and stained for p38 MAPK (red), $\gamma \mathrm{H} 2 \mathrm{AX}$ (green), and TOPRO-3 (blue). 'Merge' indicates the merged images of TOPRO-3 and p38 MAPK. A higher magnification view of the insets (marked with white boxes) in the 'merge' panels is shown on the right side. (D) Graph displaying the percentage of cells demonstrating P38 MAPK nuclear accumulation. Determined in total thymocytes from WT and $M K K 3^{-1 /} / M_{K K} 6^{+/-}$mice left unsitmulated or exposed to X-Ray (total count $>150$ cells per population, counted from 3 independent areas of visualization). Error bars represent SEM. Data representative of at least three independent experiments.

MKK3 and MKK6 are the main MAPKKs that phosphorylate and activate p38 MAPK [28]. Double deficiency for both MKK3 and MKK6 (MKK3 $\%$ - $/ \mathrm{MKK}^{-/-}$) in mice causes embryonic lethality, similar to $\mathrm{p} 38 \alpha \mathrm{MAPK}$ deficiency [8]. In contrast, MKK3 $\% /-\mathrm{MKK}^{+/-}$mice are viable, but p38 MAPK activation is severely compromised (Sabio, G. et al unpublished data). To confirm that activation of p38 MAPK was impaired in thymocytes from these mice we examined the levels of phospho-p38 MAPK by Western blot analysis. The levels of phospho-p38 MAPK were reduced in total thymocytes from MKK3 $\%$ - $/ \mathrm{MKK}^{+/-}$mice compared with the levels in wild type thymocytes (Fig 4B). We also examined the levels of activated p38 MAPK in the double negative (DN) thymocyte population where $\mathrm{p} 38$ MAPK is constitutively activated [29]. The levels of phospho-p38 MAPK were also substantially reduced in DN thymocytes from MKK3-/-/MKK6 ${ }^{+/-}$mice (Fig 4B). Thus, activation of p38 MAPK is impaired in
MKK3 $-/-/ \mathrm{MKK}^{+} /-$thymocytes. Wild type and MKK3 $\%$ - $/ \mathrm{MKK}^{+/-}$thymocytes were then exposed to $X$-radiation, and p38 MAPK intracellular distribution was assessed. As described above, X-radiation induced the nuclear accumulation of p38 MAPK in wild type total thymocytes (Fig 4C and 4D). In contrast, nuclear translocation of p38 MAPK was severely compromised in MKK3//-/MKK6 $6^{+/-}$thymocytes (Fig $4 \mathrm{C})$. The presence of DSBs, as determined by $\gamma \mathrm{H} 2 \mathrm{AX}$ staining, was comparable between exposed wild type and MKK3 $/-/ \mathrm{MKK}^{+/-}$thymocytes (Fig 4C), indicating that $\mathrm{MKK} 3^{-/-} / \mathrm{MKK}^{+/-}$thymocytes responded to $\mathrm{X}$-radiation. These results demonstrate that in primary cells, endogenous p38 MAPK translocates to the nucleus in response to DNA damage, and that nuclear translocation requires its phosphorylation.

\section{$V(D) J$ recombination mediated DSBs Induce the Nuclear Translocation of p38 MAPK.}

p38 MAPK is activated by V(D)J-mediated DSBs 
during recombination of the TCR $\beta$ gene in DN3 $\left(\mathrm{CD} 25^{+} \mathrm{CD} 44^{-}\right)$thymocytes and it is inactivated once DN3 thymocytes differentiate into DN4 (CD25CD44-) thymocytes, and DNA repair has taken place [30]. We therefore examined whether activation of p38 MAPK in response to V(D)J-mediated DSBs in DN3 thymocytes also leads to nuclear accumulation of this kinase. DN3 and DN4 thymocytes were isolated from wild type mice by cell sorting, stained for p38 MAPK and examined by confocal microscopy. p38 MAPK localized primarily in the nucleus of most DN3 thymocytes, whilst it was excluded from the nucleus in DN4 thymocytes (Fig 5A). Thus, nuclear accumulation of p38 MAPK in DN3 thymocytes correlates with the activation of p38 MAPK by $\mathrm{V}(\mathrm{D}) \mathrm{J}$-mediated DSBs in this thymocyte population. To demonstrate that the nuclear accumulation of p38 MAPK in DN3 thymocytes was caused by V(D)J-mediated DSBs, we examined the localization of p38 MAPK in DN3 thymocytes isolated from
RAG1 deficient mice since they have no V(D)J-mediated DSBs, due to the lack of the RAG1 recombinase. Minimal nuclear accumulation of p38 MAPK was detected in DN3 thymocytes from RAG1 deficient mice compared with that in wild type DN3 thymocytes (Fig 5B and 5C). Unlike RAG1-deficient thymocytes, thymocytes from SCID (Severe Combined Immuno Deficient) mice undergo V(D)J recombination, but they are unable to repair DNA damage due to a deficiency in DNA-PK. As a result, thymocyte development in these mice is also arrested at the DN3 stage, but V(D)J-mediated DNA DSBs are continuously present in these cells since they cannot be repaired. In contrast to DN3 thymocytes from RAG1 mice, most SCID DN3 thymocytes showed p38 MAPK in the nucleus (Fig 5C). Together, these results show that $\mathrm{V}(\mathrm{D}) \mathrm{J}$-mediated DSBs promote the nuclear accumulation of endogenous p38 MAPK, comparable to the response observed upon ionizing irradiation exposure.
A

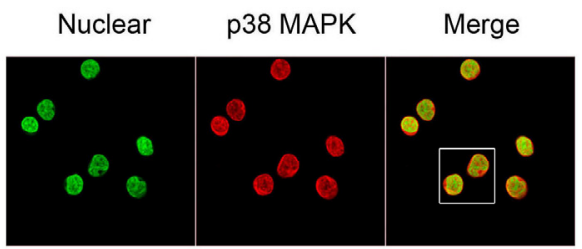

DN4

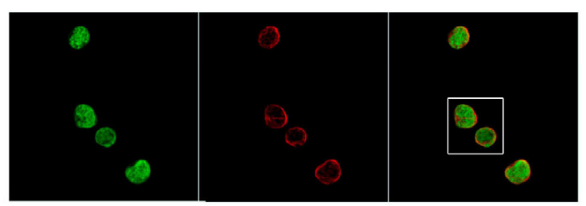

C

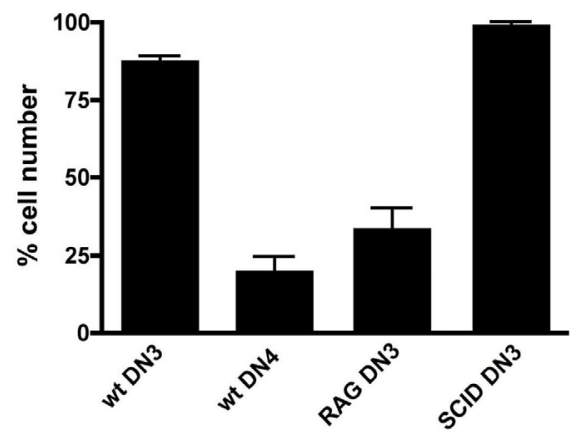

B

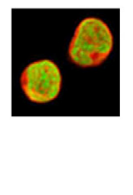

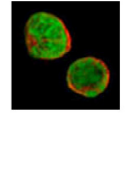

DN3
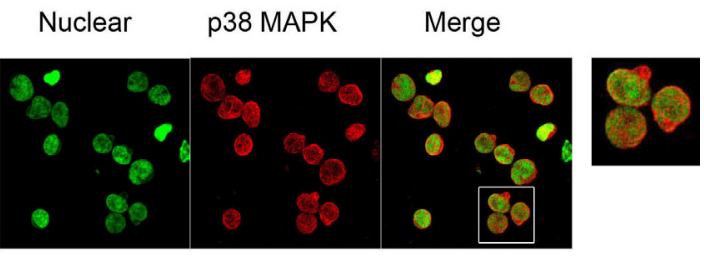

DN4
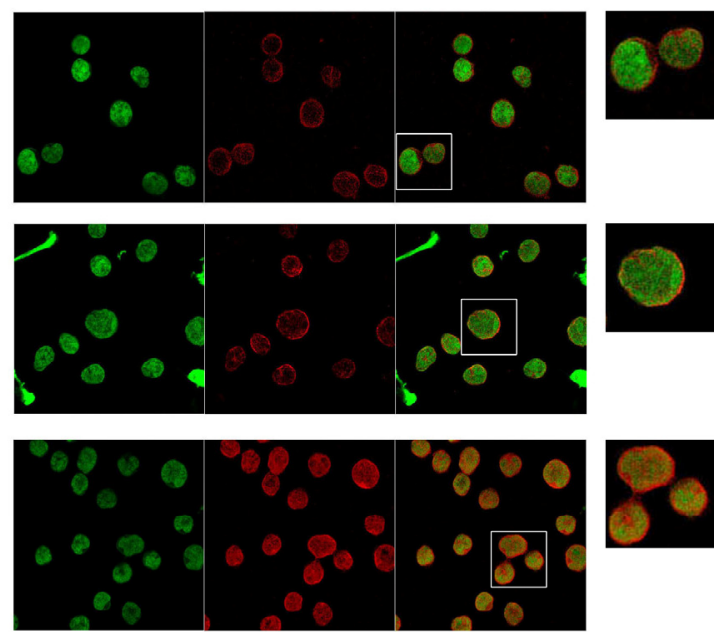

Figure 5. V(D)J-mediated DSBs Induce p38 MAPK Translocation in DN3 Thymocytes. (A) DN3 and DN4 thymocytes purified from wild type mice, were stained with for p38 MAPK (red) and YoYo (green). (B) DN3 and DN4 thymocytes purified from wild type mice and DN3 thymocytes from SCID, and RAG I- mice were stained as in (A). A higher magnification view of the insets (marked with white boxes) in the 'merge' panels is shown on the right side. Experiment representative of at least three independent experiments. (C) Graph displaying the percentage of cells demonstrating $\mathrm{p} 38$ MAPK nuclear accumulation in different cell populations (total count $>150$ cells per population, counted from 3 independent areas of visualization). Error bars represent SEM. Data representative of four experiments and multiple fields of view. 


\section{Discussion}

p38 MAPK has no nuclear localization signal, and it is believed to be diffused throughout the nucleus and the cytoplasm. In this study, we show that p38 MAPK accumulates in the nucleus specifically in response to stimuli that induce DSBs, but not by other stimuli (e.g. TNF $\alpha$, FasL) that also activate p38 MAPK. The intracellular distribution of p38 MAPK can therefore be determined by the nature of the stimuli and can influence p38 MAPK targets. Thus, DNA damage stimuli promotes nuclear accumulation of p38 MAPK for this kinase to phosphorylate potential nuclear targets (e.g. p53) involved in the induction of cell cycle checkpoints. However, arsenite treatment has been shown to promote cytoplasmic accumulation of p38 MAPK [17].

We also show here that nuclear translocation of p38 MAPK in response to DNA damage stimuli requires phosphorylation of $\mathrm{Thr}^{180} / \mathrm{Tyr}^{182}$ by MKK3 and/or MKK6, but it does not require its catalytic activity. This suggests that phosphorylation of these residues may induce a conformational change that unmasks a domain enabling p38 MAPK to interact with a nuclear shuttling chaperone to mediate its re-localization to the nucleus. To date, we cannot conclude whether phosphorylation of one or both residues is necessary for nuclear translocation. The results showing that BIRB 796, but not SB203580, restores nuclear localization of dnp38 MAPK further support the effect of a conformational change. Since phosphorylation of p38 MAPK in response to DNA damage, but not in response to other stimuli, promotes nuclear accumulation, it is possible that this nuclear shuttle is also specifically induced or activated by DNA damage, as described for NBS1 [31] and GADD45 $[32,33]$. Thus, selective nuclear transport of p38 MAPK would require both its phosphorylation as well as the active nuclear shuttle to be present. Alternatively, DNA damage signals could also release p38 MAPK from docking molecules that retain p38 MAPK in the cytosol. In this regard, p38 MAPK strongly associates with MAPKAP kinase-2 (MAPKAPK2), a substrate for p38 MAPK which contains both nuclear localization and nuclear export sequences [34]. Binding of phosphorylated MAPKAPK2 to p38 MAPK appears to promote the retention of p38 MAPK in the cytosol [17]. Similarly, binding of TAB-1 (TAK-1 binding protein) to p38 MAPK also directs localization to the cytosol, and induces p38 MAPK autophosphorylation in a MAPKK-independent manner $[35,36]$. It is plausible that the conformational change of p38 MAPK upon DNA damage disrupt its interaction with
MAPKAPK2 or TAB1, enabling its accumulation in the nucleus.

Several studies have shown a role for p38 MAPK in the induction of a G2/M cell cycle checkpoint in response to DNA damage (ionizing radiation, UV), and phosphorylation of targets involved in this checkpoint such as p53 or Cdc25 [12, 37-39]. p38 MAPK is also activated by V(D)J-mediated DSBs and induces a G2/M cell cycle checkpoint in DN3 thymocytes [30], suggesting that V(D)J-mediated DSBs also represent DNA damage signals. We show here nuclear accumulation of p38 MAPK specifically in DN3 thymocytes undergoing $\mathrm{V}(\mathrm{D}) \mathrm{J}$ recombination (wild type and SCID), but not in DN3 thymocytes from RAG deficient mice. Thus, similar to the DSBs induced by ionizing radiation, $\mathrm{V}(\mathrm{D}) \mathrm{J}$-mediated DSBs appear to be interpreted as a DNA damage signal. Recent studies have shown that signal ended DNA fragments released during $\mathrm{V}(\mathrm{D}) \mathrm{J}$ recombination of the TCR $\beta$ gene can integrate into different chromosomal locations, by either insertion or transposition [40-45], and may lead to genomic instability and potential malignancies. The selective nuclear accumulation of p38 MAPK in response to DNA damage could pose a mechanism to facilitate the phosphorylation of p38 MAPK nuclear targets for the induction of a G2/M cell cycle checkpoint and DNA repair.

In summary, the intracellular localization of p38 MAPK is regulated upon activation of this kinase, and is dependent on the stimuli involved. This most likely reflects the requirement of p38 MAPK to be in close proximity to its targets.

\section{Materials and Methods}

\section{Mice.}

Wild type, RAG1 $\%$, and SCID mice were purchased from Jackson Laboratories. MKK3 $/$-and MKK $6^{-/}$- single mice have been previously reported [46]. MKK3 $/-/ \mathrm{MKK}^{+/-}$were obtained by serial intercrosses of $\mathrm{MKK}^{-/}$and $\mathrm{MKK} 6 \%$ single mice as previously reported [8]. Mice (five to seven weeks of age) were bred and maintained in specific pathogen-free conditions. Procedures involving mice were approved by institutional guidelines for animal care.

\section{Cell Preparation, Transfection, and Stimulation.}

HEK293T cells were transfected using calcium phosphate. Following exposure to UV (1.5 mins, 15 $\mathrm{J} / \mathrm{m}^{2}$ ), or X-radiation (2.8 Gy) (RadSource 2000, GA) cells were rested at $37^{\circ} \mathrm{C}$ for 30 mins. SB203580 (Calbiochem, CA) and BIRB 796 (Axon Medchem) pretreatment $(5 \mu \mathrm{M})$ was performed at $37^{\circ} \mathrm{C}$ for 40 mins, prior to cell stimulation. Cells were additionally stimulated with PMA (Sigma) $(10 \mathrm{ng} / \mathrm{ml}), \mathrm{TNF} \alpha$ 
(R\&D Systems, MN) $(10 \mathrm{ng} / \mathrm{ml})$, or Ig-FasL (Alexis Biochemicals, CA) $(200 \mathrm{ng} / \mathrm{ml})$ and anti-Flag M2 mouse monoclonal antibody (Sigma) $(4 \mu \mathrm{g} / \mathrm{ml})$ at $37^{\circ} \mathrm{C}$ for 30 mins, prior to fixation. DN3 and DN4 thymocytes were purified and stained (CD4, CD8, CD25, and CD44 BD Biosciences), sorted (FacsAria, BD Biosciences), and collected.

\section{Confocal Microscopy and Analysis.}

Staining of 293T [47] cells and thymocytes [30] was performed as we previously published. Briefly, cells were fixed, permeabilized and stained with primary antibody followed by fluorescently labeled secondary antibody. The primary antibodies used included the rabbit anti-p38 MAPK polyclonal antibody (Santa Cruz, CA), mouse anti- $\gamma \mathrm{H} 2 \mathrm{AX}$ monoclonal antibody (Upstate, Millipore), rabbit anti-phospho-p38 MAPK monoclonal antibody \#9215 recognizing p38 MAPK dually phosphorylated on $\mathrm{Thr}^{180} / \mathrm{Tyr}^{182}$ (Cell Signaling, MA). Secondary antibodies used include Alexa Fluor anti-mouse/anti-rabbit 488/567 highly adsorbed antibodies (Invitrogen). Topro-3 and YoYo were used as nuclear stains. Images of fixed cells were acquired on a Zeiss LSM-510 upright microscope using a 63x Plan Apochromat Ph3 objective lens.

\section{Western Blot.}

Whole cell extracts were prepared in Triton lysis buffer (TLB) and used for Western blot analysis as we previously described [30] using anti-p38 MAPK (Santa Cruz, CA) and anti-phospho-p38 MAPK (Cell Signaling, MA). Anti-rabbit HRP, and anti-mouse HRP (Santa Cruz, CA) were used as secondary antibodies.

\section{Acknowledgements}

We would like to thank Douglas Taatjes and Marilyn Wadsworth at the UVM Microscopy Imaging Center. This work was supported by a NIH R01 A1051454 grant (M.R.).

\section{Conflict of Interest}

The authors have declared that no conflict of interest exists.

\section{References}

1. Adams R.H., Porras A., Alonso G., Jones M., Vintersten K., Panelli S., Valladares A., Perez L., Klein R., and Nebreda A.R. Essential role of p38alpha MAP kinase in placental but not embryonic cardiovascular development. Mol Cell 2000;6: 109-116.

2. Allen M., Svensson L., Roach M., Hambor J., McNeish J., and Gabel C.A. Deficiency of the stress kinase p38alpha results in embryonic lethality: characterization of the kinase dependence of stress responses of enzyme-deficient embryonic stem cells. J Exp Med 2000;191: 859-870.

3. Mudgett J.S., Ding J., Guh-Siesel L., Chartrain N.A., Yang L., Gopal S., and Shen M.M. Essential role for p38alpha mitogen-activated protein kinase in placental angiogenesis. Proc Natl Acad Sci U S A 2000;97: 10454-10459.

4. Tamura K., Sudo T., Senftleben U., Dadak A.M., Johnson R., and Karin M. Requirement for p38alpha in erythropoietin expression: a role for stress kinases in erythropoiesis. Cell 2000;102: 221-231.

5. Beardmore V.A., Hinton H.J., Eftychi C., Apostolaki M., Armaka M., Darragh J., McIlrath J., Carr J.M., Armit L.J., Clacher C., et al. Generation and characterization of p38beta (MAPK11) gene-targeted mice. Mol Cell Biol 2005;25: 10454-10464.

6. Derijard B., Raingeaud J., Barrett T., Wu I.H., Han J., Ulevitch R.J., and Davis R.J. Independent human MAP-kinase signal transduction pathways defined by MEK and MKK isoforms. Science 1995;267: 682-685.

7. Han J., Lee J.D., Jiang Y., Li Z., Feng L., and Ulevitch R.J. Characterization of the structure and function of a novel MAP kinase kinase (MKK6). J Biol Chem 1996;271: 2886-2891.

8. Brancho D., Tanaka N., Jaeschke A., Ventura J.J., Kelkar N., Tanaka Y., Kyuuma M., Takeshita T., Flavell R.A., and Davis R.J. Mechanism of p38 MAP kinase activation in vivo. Genes Dev 2003;17: 1969-1978.

9. Zarubin T., and Han J. Activation and signaling of the p38 MAP kinase pathway. Cell research 2005;15: 11-18.

10. She Q.B., Chen N., and Dong Z. ERKs and p38 kinase phosphorylate p53 protein at serine 15 in response to UV radiation. J Biol Chem 2000;275: 20444-20449.

11. She Q.B., Bode A.M., Ma W.Y., Chen N.Y., and Dong Z. Resveratrol-induced activation of p53 and apoptosis is mediated by extracellular-signal-regulated protein kinases and p38 kinase. Cancer Res 2001;61: 1604-1610.

12. Bulavin D.V., Higashimoto Y., Popoff I.J., Gaarde W.A., Basrur V., Potapova O., Appella E., and Fornace AJJr. Initiation of a G2/M checkpoint after ultraviolet radiation requires p38 kinase. Nature 2001;411: 102-107.

13. Mikhailov A., Shinohara M., and Rieder C.L. The p38-mediated stress-activated checkpoint. A rapid response system for delaying progression through antephase and entry into mitosis. Cell Cycle 2005;4: 57-62.

14. Kurosu T., Takahashi Y., Fukuda T., Koyama T., Miki T., and Miura O. p38 MAP kinase plays a role in G2 checkpoint activation and inhibits apoptosis of human B cell lymphoma cells treated with etoposide. Apoptosis 2005;10: 1111-1120.

15. Thornton T.M., and Rincon M. Non-classical p38 map kinase functions: cell cycle checkpoints and survival. Int J Biol Sci 2009;5: 44-51.

16. Raingeaud J., Gupta S., Rogers J.S., Dickens M., Han J., Ulevitch R.J., and Davis R.J. Pro-inflammatory cytokines and environmental stress cause p38 mitogen-activated protein kinase activation by dual phosphorylation on tyrosine and threonine. J Biol Chem 1995;270: 7420-7426.

17. Ben-Levy R., Hooper S., Wilson R., Paterson H.F., and Marshall C.J. Nuclear export of the stress-activated protein kinase p38 mediated by its substrate MAPKAP kinase-2. Curr Biol 1998;8: 1049-1057.

18. Cuenda A., Cohen P., Buee-Scherrer V., and Goedert M. Activation of stress-activated protein kinase-3 (SAPK3) by cytokines and cellular stresses is mediated via SAPKK3 (MKK6); comparison of the specificities of SAPK3 and SAPK2 (RK/p38). EMBO J 1997;16: 295-305.

19. Waskiewicz A.J., Flynn A., Proud C.G., and Cooper J.A. Mitogen-activated protein kinases activate the serine/threonine kinases Mnk1 and Mnk2. EMBO J 1997;16: 1909-1920. 
20. Zhao M., New L., Kravchenko V.V., Kato Y., Gram H., di Padova F., Olson E.N., Ulevitch R.J., and Han J. Regulation of the MEF2 family of transcription factors by p38. Mol Cell Biol 1999;19: 21-30.

21. Alvarado-Kristensson M., Melander F., Leandersson K., Ronnstrand L., Wernstedt C., and Andersson T. p38-MAPK signals survival by phosphorylation of caspase- 8 and caspase- 3 in human neutrophils. J Exp Med 2004;199: 449-458.

22. Trouillas M., Saucourt C., Duval D., Gauthereau X., Thibault C., Dembele D., Feraud O., Menager J., Rallu M., Pradier L., et al. Bcl2, a transcriptional target of p38alpha, is critical for neuronal commitment of mouse embryonic stem cells. Cell Death Differ 2008;15: 1450-1459.

23. Jenner T.J., Fulford J., and O'Neill P. Contribution of base lesions to radiation-induced clustered DNA damage: implication for models of radiation response. Radiation research 2001;156: 590-593.

24. Rogakou E.P., Pilch D.R., Orr A.H., Ivanova V.S., and Bonner W.M. DNA double-stranded breaks induce histone H2AX phosphorylation on serine 139. J Biol Chem 1998;273: 5858-5868.

25. Sedelnikova O.A., Rogakou E.P., Panyutin I.G., and Bonner W.M. Quantitative detection of (125)IdU-induced DNA double-strand breaks with gamma-H2AX antibody. Radiation research 2002;158: 486-492.

26. Kumar S., McDonnell P.C., Gum R.J., Hand A.T., Lee J.C., and Young P.R. Novel homologues of CSBP/p38 MAP kinase: activation, substrate specificity and sensitivity to inhibition by pyridinyl imidazoles. Biochem Biophys Res Commun 1997;235: 533-538.

27. Pargellis C., Tong L., Churchill L., Cirillo P.F., Gilmore T., Graham A.G., Grob P.M., Hickey E.R., Moss N., Pav S., et al. Inhibition of p38 MAP kinase by utilizing a novel allosteric binding site. Nat Struct Biol 2002;9: 268-272.

28. Enslen H., Raingeaud J., and Davis R.J. Selective activation of p38 mitogen-activated protein (MAP) kinase isoforms by the MAP kinase kinases MKK3 and MKK6. J Biol Chem 1998;273: 1741-1748.

29. Sen J., Kapeller R., Fragoso R., Sen R., Zon L.I., and Burakoff S.J. Intrathymic signals in thymocytes are mediated by p38 mitogen-activated protein kinase. J Immunol 1996;156: 4535-4538.

30. Pedraza-Alva G., Koulnis M., Charland C., Thornton T., Clements J.L., Schlissel M.S., and Rincon M. Activation of p38 MAP kinase by DNA double-strand breaks in V(D)J recombination induces a G2/M cell cycle checkpoint. Embo J 2006;25: 763-773.

31. Carney J.P., Maser R.S., Olivares H., Davis E.M., Le Beau M., Yates JR3rd, Hays L., Morgan W.F., and Petrini J.H. The hMre11/hRad50 protein complex and Nijmegen breakage syndrome: linkage of double-strand break repair to the cellular DNA damage response. Cell 1998;93: 477-486.

32. Hollander M.C., Alamo I., Jackman J., Wang M.G., McBride O.W., and Fornace AJJr. Analysis of the mammalian gadd45 gene and its response to DNA damage. J Biol Chem 1993;268: 24385-24393.

33. Zhu N., Shao Y., Xu L., Yu L., and Sun L. Gadd45-alpha and Gadd45-gamma utilize p38 and JNK signaling pathways to induce cell cycle G2/M arrest in Hep-G2 hepatoma cells. Mol Biol Rep. 2008 [Epub ahead of print]

34. Engel K., Kotlyarov A., and Gaestel M. Leptomycin B-sensitive nuclear export of MAPKAP kinase 2 is regulated by phosphorylation. EMBO J 1998;17: 3363-3371.

35. Ge B., Gram H., Di Padova F., Huang B., New L., Ulevitch R.J., Luo Y., and Han J. MAPKK-independent activation of p38alpha mediated by TAB1-dependent autophosphorylation of p38alpha. Science 2002;295: 1291-1294.

36. Lu G., Kang Y.J., Han J., Herschman H.R., Stefani E., and Wang Y. TAB-1 modulates intracellular localization of p38 MAP kinase and downstream signaling. J Biol Chem 2006;281: 6087-6095.

37. Dmitrieva N.I., Bulavin D.V., Fornace AJJr., and Burg M.B. Rapid activation of G2/M checkpoint after hypertonic stress in renal inner medullary epithelial (IME) cells is protective and requires p38 kinase. Proc Natl Acad Sci U S A 2002;99: 184-189.

38. Chao J.I., and Kuo P.C. Role of p53 and p38 MAP kinase in nitric oxide-induced $\mathrm{G} 2 / \mathrm{M}$ arrest and apoptosis in the human lung carcinoma cells. Carcinogenesis 2004;25: 645.

39. Reinhardt H.C., Aslanian A.S., Lees J.A., and Yaffe M.B. p53-deficient cells rely on ATM- and ATR-mediated checkpoint signaling through the p38MAPK/MK2 pathway for survival after DNA damage. Cancer cell 2007;11: 175-189.

40. Messier T.L., O'Neill J.P., and Finette B.A. V(D)J recombinase mediated inter-chromosomal HPRT alterations at cryptic recombination signal sequences in peripheral human $\mathrm{T}$ cells. Hum Mutat 2006;27: 829.

41. Murray J.M., O'Neill J.P., Messier T., Rivers J., Walker V.E., McGonagle B., Trombley L., Cowell L.G., Kelsoe G., McBlane F., et al. V(D)J recombinase-mediated processing of coding junctions at cryptic recombination signal sequences in peripheral $\mathrm{T}$ cells during human development. J Immunol 2006;177: 5393-5404.

42. Chatterji M., Tsai C.L., and Schatz D.G. Mobilization of RAG-generated signal ends by transposition and insertion in vivo. Mol Cell Biol 2006;26: 1558-1568.

43. Reddy Y.V., Perkins E.J., and Ramsden D.A. Genomic instability due to $V(D) J$ recombination-associated transposition. Genes Dev 2006;20: 1575-1582.

44. Vanura K., Montpellier B., Le T., Spicuglia S., Navarro J.M., Cabaud O., Roulland S., Vachez E., Prinz I., Ferrier P., et al. In vivo reinsertion of excised episomes by the $\mathrm{V}(\mathrm{D}) \mathrm{J}$ recombinase: a potential threat to genomic stability. PLoS biology 2007;5: e43.

45. Curry J.D., Schulz D., Guidos C.J., Danska J.S., Nutter L., Nussenzweig A., and Schlissel M.S. Chromosomal reinsertion of broken RSS ends during $\mathrm{T}$ cell development. J Exp Med 2007;204: 2293-2303.

46. Tanaka N., Kamanaka M., Enslen H., Dong C., Wysk M., Davis R.J., and Flavell R.A. Differential involvement of p38 mitogen-activated protein kinase kinases MKK3 and MKK6 in T-cell apoptosis. EMBO Rep 2002;3: 785-791.

47. Hatle K.M., Neveu W., Dienz O., Rymarchyk S., Barrantes R., Hale S., Farley N., Lounsbury K.M., Bond J.P., Taatjes D., et al. Methylation-controlled J protein promotes c-Jun degradation to prevent ABCB1 transporter expression. Mol Cell Biol 2007;27: 2952-2966. 